\title{
Modeling Control Strategies of Respiratory Pathogens
}

\author{
Babak Pourbohloul,*1 Lauren Ancel Meyers, $\dagger^{1}$ Danuta M. Skowronski, ${ }^{*}$ Mel Krajden, ${ }^{*}$ \\ David M. Patrick, ${ }^{*}$ and Robert C. Brunham*
}

Effectively controlling infectious diseases requires quantitative comparisons of quarantine, infection control precautions, case identification and isolation, and immunization interventions. We used contact network epidemiology to predict the effect of various control policies for a mildly contagious disease, such as severe acute respiratory syndrome, and a moderately contagious disease, such as smallpox. The success of an intervention depends on the transmissibility of the disease and the contact pattern between persons within a community. The model predicts that use of face masks and general vaccination will only moderately affect the spread of mildly contagious diseases. In contrast, quarantine and ring vaccination can prevent the spread of a wide spectrum of diseases. Contact network epidemiology can provide valuable quantitative input to public health decisionmaking, even before a pathogen is well characterized.

$\mathrm{P}$ ublic concern regarding emerging infectious diseases is on the rise. The 21st century began with the emergence or reemergence of zoonotic diseases like severe acute respiratory syndrome (SARS) (1), avian influenza (2), monkeypox infection (3), West Nile virus disease (4), mad cow disease (5), anthrax due to bioterrorist attacks (6), and unusual influenza epidemics (7). In addition to these new threats, public health officials face a large number of disease outbreaks every year in hospitals, schools, and other small communities. While development of vaccines and diagnostic tools proceeds at an unprecedented pace, development of tools for determining optimal intervention strategies lags behind.

In response to this problem, we have found that mathematical models of disease transmission can be used to

*University of British Columbia Centre for Disease Control, Vancouver, British Columbia, Canada; †University of Texas at Austin, Austin, Texas, USA; and ‡Santa Fe Institute, Santa Fe, New Mexico, USA evaluate and optimize control strategies. Such quantitative predictions can be empirically tested through randomized comparative trials, and mathematical models increasingly contribute to public health decisions regarding policy and intervention (8-13).

We use contact network epidemiology to compare intervention strategies for airborne ${ }^{2}$ infectious diseases, including emerging diseases such as SARS, for which epidemiologic data are limited. These methods are based on explicit mathematical models of the heterogeneous patterns of interpersonal contacts that underlie disease transmission in a community, be it a hospital, school, or city (12-21). This approach differs from fully mixed compartmental models that assume that each person can infect every other person with equal probability (8). Some compartmental models have been modified to include population heterogeneity and have provided insights into the long-term effects of intervention strategies (8-11). For communities with extensive heterogeneity in contact patterns, however, network models more explicitly capture patterns of disease transmission and thus enable more accurate and detailed predictions of the effect of control measures on the magnitude and distributions of outbreaks.

\section{Methods}

Contact network models capture and estimate interpersonal contacts that lead to disease transmission within a community (22). Contacts can take place within households, schools, workplaces, hospitals, and other public venues. Each person in a community is represented as a

\footnotetext{
${ }^{1}$ These authors contributed equally to this work.
}

2For the purposes of this manuscript, "airborne" refers to respiratory pathogens that are spread through respiratory secretions and can be either airborne, such as tuberculosis, or dropletborne, such as SARS. 
vertex in the network, and each contact between 2 people is represented as an edge connecting the vertexes. The number of edges emanating from a vertex is the degree of that vertex. This quantity indicates the number of contacts who potentially transmit disease to or acquire disease from a person. The variation of degree across the entire network, i.e., the degree distribution, is fundamental to determining the probability for spread of disease through a network of contacts. Given the degree distribution of the contact network, one can analytically predict the fate of an outbreak.

Contact network epidemiology allows us to assess the vulnerability of a population to an infectious disease on the basis of the structure of the network (its degree distribution) and on the average transmissibility $(T)$ of the disease $(12,13) . T$ is the average probability that transmission will occur from an infected person (vertex) to an uninfected person. This parameter summarizes multiple aspects of transmissibility, including the contact intensity between persons, duration of infectiousness, and the host's susceptibility to the infectious pathogen $(12,13)$.

\section{Contact Network Parameter Estimation}

We built an urban contact network model with 2,000 households with an average household size of 2.6 (5,154 persons) based on demographic information for the Greater Vancouver Regional District, British Columbia, Canada. We used publicly available data from sources such as Statistics Canada to estimate the distribution of ages, household sizes, school and classroom sizes, hospital occupancy, workplaces, and public spaces (23-27).

Most of the edges in the network are undirected, meaning that transmission may occur in either direction (black edges in Figure 1). For example, 2 persons living in the same household will have equal opportunities to infect each other. The remaining edges are directed, meaning that a person may infect another person but the converse is not true (gray edges in Figure 1). For example, suppose person A is healthy and has no reason to go to the hospital until he or she is infected with SARS. At that point, person A will likely come into contact with and potentially spread SARS to caregivers at the hospital. In contrast, if a caregiver at the hospital acquired SARS while person A remained healthy in the community, then no opportunity would exist for transmission in the opposite direction. To model the directional flow of infected patients into a hospital, we include directed edges from persons in the population at large to caregivers in the hospital.

In an urban setting, not all encounters are equally likely to lead to disease transmission. We capture this heterogeneity in 2 ways. First, in the simulated urban network, the probability of a contact between 2 persons depends on the location and nature of their overlapping daily activities. For example, persons in the same household are connected

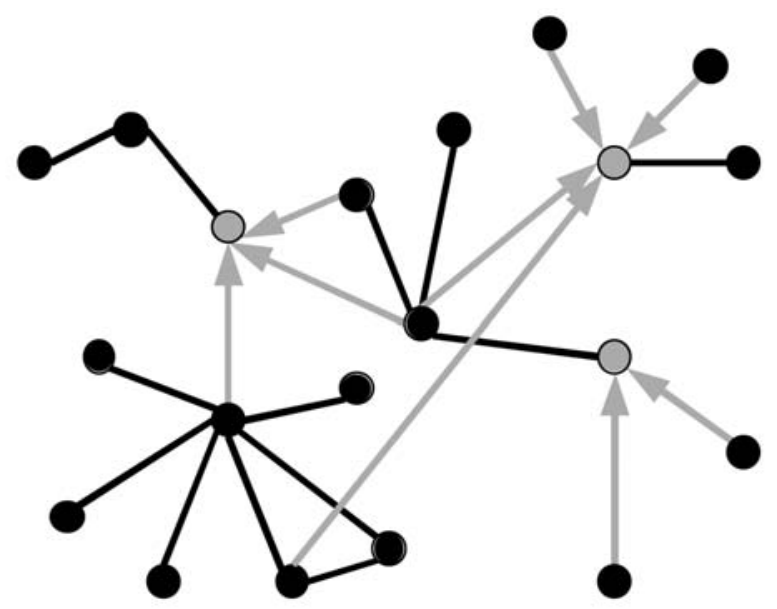

Figure 1. Schematic diagram of a directed network. Each black vertex represents a member of the general population; gray vertexes represent healthcare workers.

to each other with probability 1 , while persons who encounter each other in a public space are connected to each other with a probability from 0.003 to 0.300 . Second, after these connections are determined, we assign a distinct transmissibility, $T_{i j}$, for each pair of connected persons $i$ and $j$, that depends on the nature of their contact. For a given disease, the distribution of transmissibilities is based on empiric estimates for the diversity in infectious periods and the per day probability of transmission between persons who come into contact with each other. For more details, please refer to the online Appendix, available from http://www.cdc.gov/ncidod/EID/vol11no08/04-0449_ app.htm.

\section{Modeling Control Strategies}

In any given network exists a critical transmissibility value, $T_{c}$, which indicates whether a large-scale epidemic is probable. Any disease with average transmissibility $<T_{c}$ cannot cause sustained transmission within a population and will thus be limited to small outbreaks. Such diseases die out because of the probabilistic nature of transmission before the disease has a chance to spread to the population at large. In this case, we can mathematically predict the expected size of small outbreaks, $s$. Diseases with average transmissibility $>T_{c}$ will spark large-scale epidemics with probability $S$, which can also be estimated. The value of $T_{c}$ depends on the contact patterns within a community. Roughly speaking, when abundant opportunities exist for transmission, disease will spread easily, and the epidemic threshold will be low. The equations for $s, S$, and $T_{c}$, which are entirely in terms of the degree distribution and average transmissibility $T$, are presented in the online Appendix.

The epidemic potential of disease is commonly estimated by using the basic reproductive number $R_{0}$, the number 
of secondary infections arising from a single infection in a relatively naïve population $(8,28)$. This quantity is linearly related to the transmissibility of the disease, i.e., $R_{0}=\gamma T$, where $\gamma$ depends on the structure of the network (equations 1 and 8 in online Appendix). When $T$ is at the epidemic threshold $\left(T=T_{c}\right.$ ), then $R_{0}=1$. Public health interventions aim to reduce the number of new infected cases, ideally decreasing the effective reproductive number of the disease below the epidemic threshold, $R_{\text {eff }}<1$.

The difference between average transmissibility $T$ and the basic reproductive ratio $R_{0}$ is important. While both have threshold values that distinguish epidemic from nonepidemic scenarios $\left(R_{0}=1\right.$ and $\left.T=T_{c}\right)$, $T$ is determined by the transmission characteristics of the pathogen and the nature of human interactions, but not the numbers of contacts in a community, whereas $R_{0}$ depends on all of these factors, particularly on the numbers of interactions within the community. For example, consider a single airborne pathogen spreading through a hospital, where abundant close contacts exist, and through a rural community, where close contacts are rare. The per contact probabilities of transmission $\left(T_{i j}\right)$ may be similar in these settings because they are determined by the pathogenesis of the strain in the host, while the numbers of contacts are different. Therefore, the average transmissibility $T$ will be similar in the 2 locations, while $R_{0}$ will be substantially higher in the hospital than in the rural setting.

The heterogeneous spread of SARS worldwide suggested context-dependent patterns of transmission with relatively rapid spread through hospitals and relatively slow spread through communities (29). A notable exception to this pattern, the large cluster of SARS cases outside a healthcare setting in the Amoy Gardens apartment complex in Hong Kong, seems to have spread through aerosolization of virus-laden sewage rather than direct person-to-person contact (30). When contact patterns within a community are extremely heterogeneous, explicitly modeling community structure and $T$ makes more sense than assuming a universal $R_{0}$. We take this approach to evaluating disease control strategies in an urban setting (31).

A primary public health goal is to bring disease from a value above an epidemic threshold to a value below the threshold, thereby eliminating the threat of a large-scale epidemic. This goal can be achieved through interventions that directly affect the transmissibility of the pathogen $(T)$ or through interventions that modify patterns of interaction so that the epidemic threshold $\left(T_{c}\right)$ is increased. We call these 2 forms of intervention transmission-reduction and contact-reduction, respectively, and depict them graphically in Figure 2. The solid curves represent the predicted size of an outbreak and the probability of an epidemic for an entire spectrum of $T$ from 0 to 1 in an urban setting. All airborne pathogens have a transmissibility value within this
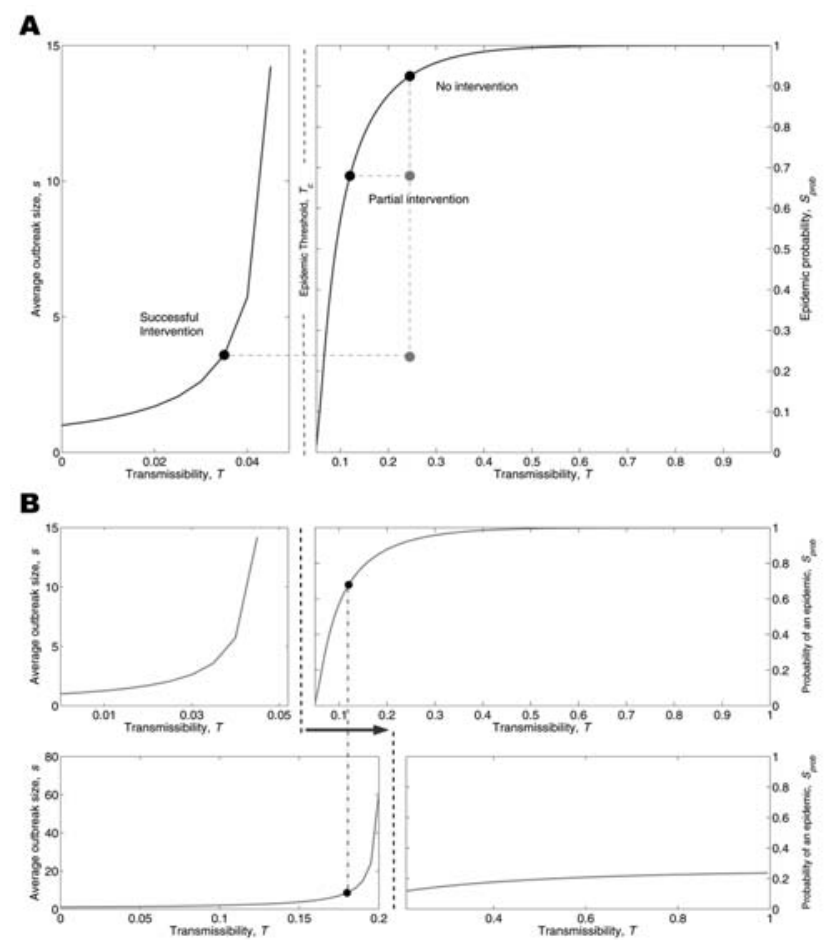

Figure 2. Transmission- vs. contact-reduction intervention. A) Transmission-reduction intervention: solid curves show the average size of an outbreak (left panel) and the probability of a largescale epidemic (right panel). The horizontal axes cover the spectrum of disease transmissibility (from 0 to 1 ) such that a single disease is associated with a unique value on either the left curve (if $T<T_{c}$ ) or the right curve (if $T>T_{c}$ ). The epidemic threshold $T_{c}$ separates the 2 zones. For better visualization, we chose 2 different scales for horizontal axes of the 2 panels. Consider a disease with $T=0.245$ (top black circle). A transmission-reduction intervention causes the black circle to slide on a new position on the curve. A successful intervention is the one that lowers $T$ to a value $<T_{c}$. B) Contact-reduction intervention: solid curves in the top panel show the epidemiologic vulnerability of the original network. Contact-reduction interventions alter the structure of the contact network and shift the epidemic curves to the right (solid curves in bottom panel). The 2 dashed vertical lines show the critical transmissibility threshold for the old (left) and new (right) networks. Consider the disease denoted by the black circle: the contact-reduction intervention raised the epidemic threshold above transmissibility of the disease and thereby eliminated the possibility of an epidemic.

range; $0=$ no transmission, and $1=$ every contact leads to transmission. Thus, any disease can be mapped to a unique value on the curve.

In our simulated urban contact network, the critical transmissibility threshold is $T_{c}=0.048$. An outbreak of disease with $T=0.245$ will almost certainly spark an epidemic in the absence of intervention (top circle in Figure 2). This value of $T$ is equivalent to an $R_{0}=5$ for this contact network and thus corresponds to a moderately infectious disease like smallpox $(32,33)$. A successful intervention 
either reduces $T$ so that it lies below $T_{c}$ (Figure 2A) or modifies the structure of the network so that $T_{c}$ rises above $T$ (Figure 2B). The first strategy can be achieved by interventions that reduce the probability of transmission per contact, such as face masks, gloves, gowns, handwashing, and other infection control precautions that prevent the exchange of respiratory droplets without eliminating contact.

The second strategy involves modifying the contact network itself. Interventions such as quarantine and closing schools and other public places effectively eliminate potential contacts (edges) between persons. Interventions such as immunization and the prophylactic use of antibacterial or antiviral drugs are tantamount to removing persons (vertexes) from the contact network and therefore also alter the network structure. We mathematically assess the effect of such strategies by deleting edges and vertexes from the contact network and predicting the new probability of an epidemic and expected distribution of cases within the community.

\section{Results}

We evaluated a variety of commonly implemented public health interventions by changing the contact patterns within the network, transmissibility of the disease, or both. For each strategy, we calculated several epidemiologic quantities: 1) the epidemic threshold, $T_{c}$, which may be raised by contact-reduction interventions, 2) the transmissibility of the disease, $T$, which may be reduced by transmission-reduction interventions, 3) if $T<T_{c}$, the expected size of a small outbreak, $s, 4)$ if $T>T_{c}$, the probability of a large-scale epidemic, $S_{\text {prob }}$, and 5) if $T>T_{c}$, the expected size of an epidemic, $S$, should one occur. Based on calculations of these quantities, Figures 3-5 report the effect of various interventions applied to a moderately contagious disease just above the epidemic threshold (left panel), where we believe SARS to lie (34) and a moderately infectious disease such as smallpox (right panel). Gray entries correspond to unsuccessful interventions; white entries indicate strategies that are predicted to successfully move the pathogen below the epidemic threshold and thereby prevent a large-scale epidemic.

\section{Transmission Reduction}

Although general use of face masks may have a moderate effect, its success hinges on correct use and level of compliance. For instance, face masks that are $75 \%$ effective will only prevent a large-scale epidemic of a SARSlike disease if $\geq 60 \%$ of the general population complies perfectly (Figure 3). If persons use face masks incorrectly or only partially, this intervention will be less likely to protect persons and the population as a whole. For moderately contagious diseases like smallpox, face masks alone will not protect large urban areas from an epidemic. Figure 3

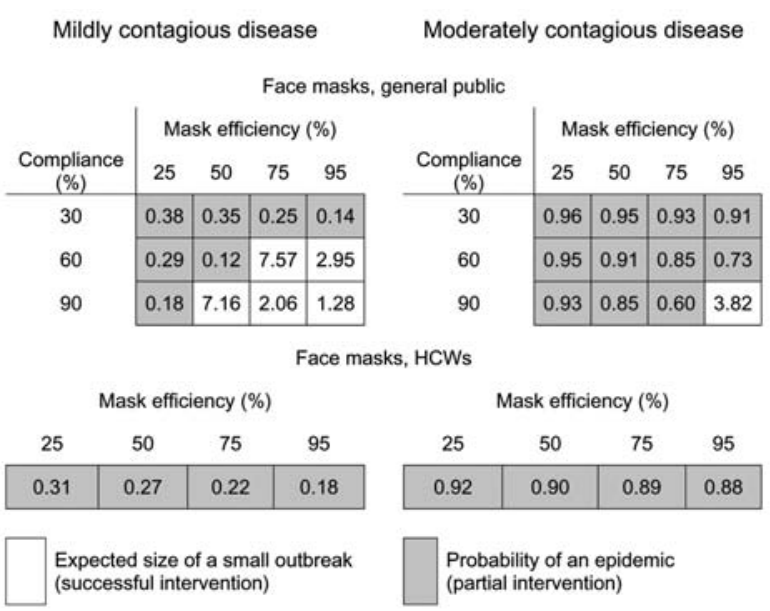

Figure 3. Comparing the effect of face masks for the general public and healthcare workers (HCWs). Mask efficiency is the percent reduction in transmissibility to or from a person correctly using a mask. Compliance is the fraction of the population adopting the intervention. Results are for a mildly contagious disease with a transmissibility $T=0.075$ and a moderately contagious disease with a transmissibility $T=0.245$. The equivalent basic reproductive number for these diseases are $R_{0}=1.545$ and $R_{0}=5.047$, respectively. Without intervention, both of these diseases have $T$ above the epidemic threshold for the community $\left(T_{c}=0.048\right)$ and thus may ignite a large-scale epidemic. The probabilities that such epidemics will occur (without intervention) are $S_{\text {prob }}=0.50$ and $S_{\text {prob }}=$ 0.97 , respectively. Some interventions may not bring $T$ below the epidemic threshold and thus only reduce the probability of an epidemic (gray boxes), while others succeed in containing transmission to a small outbreak (white boxes). Gray boxes give the probability of an epidemic, and white boxes give the expected size of an outbreak. Outbreak size may not be an integer since $s$ is an average taken from all possible outbreaks in the community.

also suggests that use of face masks by healthcare workers, while important for personal protection, offers limited protection to the population and does not predictably preclude an epidemic.

One of the factors that influences the transmissibility $T$ is the duration of infectiousness. The duration of effective infectiousness may be shortened, but not eliminated, by isolating persons immediately after diagnosis. Although isolating an infected person will physically remove him from the network, the person may already have had a chance to infect others before being identified and isolated. For example, an infectious person who is isolated after the second day of a 6-day infectious period will have had 2 days in which disease could be transmitted to close contacts. Thus, isolation can be effective for diseases with low transmissibility but only if case identification occurs early in the infectious period. For such diseases, an isolation strategy that on average reduces the infectious period by $50 \%$ will prevent a large-scale epidemic (Figure 4). Isolation will not preclude an epidemic for a highly trans- 


\begin{tabular}{l|c|c|c|c|c|c|c|c|}
\multicolumn{3}{c}{ Mildly contagious disease } & \multicolumn{5}{c}{ Moderately contagious disease } \\
\multicolumn{1}{c|}{ Isolation } \\
Infectiousness reduction (\%) & \multicolumn{5}{c}{ Infectiousness reduction (\%) } \\
\hline 25 & 50 & 75 & 95 & 25 & 50 & 75 & 95 \\
\hline 0.22 & 4.73 & 1.66 & 1.09 \\
\hline
\end{tabular}

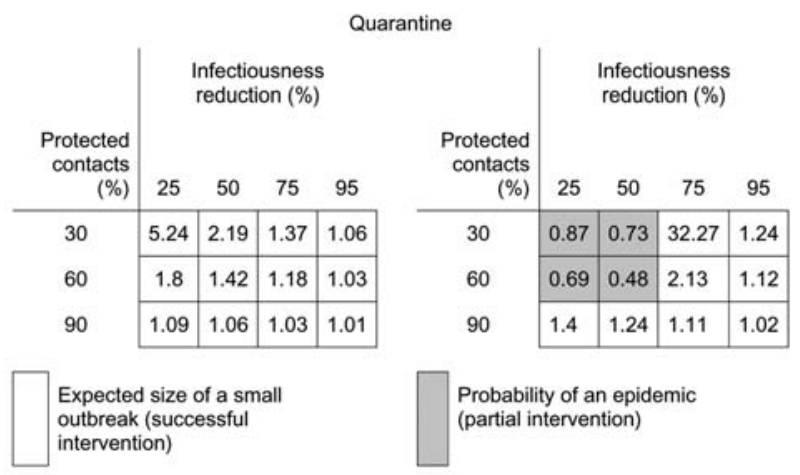

Figure 4. Comparing the effect of isolation and quarantine. Isolation alone reduces the infectious period by a specified percentage. Quarantine involves both isolation and sequestering a fraction of all case contacts. See the Figure 3 caption for further details.

missible disease unless clinical and diagnostic tools can be applied early and confidently, which may not be the case for an emerging infectious disease.

\section{Contact Reduction}

Contacts between infected and susceptible persons can be eliminated during an outbreak through measures such as quarantine, closing public venues, and ring vaccination, or they can be eliminated preventatively through general vaccination strategies. Figure 4 predicts that simultaneous case-patient isolation and quarantine of close contacts substantially improves containment. For a mildly contagious disease, an outbreak can be controlled with a combination of isolation that reduces the infectious period by $25 \%$ and quarantine that successfully sequesters $30 \%$ of all casepatient contacts. Much more rigorous isolation and quarantine are required for a highly contagious disease. Such interventions require a strong surveillance infrastructure, reliable rapid diagnostic tests, and social acceptance.

In Figure 6, we show that such predictions can readily be translated into values of $R_{\text {eff }}$ Interventions that bring a population under the epidemic threshold are those that decrease $R_{\text {eff }}$ below 1 . We emphasize that the predictions in Figures 4 and 6 are specific to the underlying model of contact patterns in an urban setting and that, contrary to common interpretations, $R_{\text {eff }}$ (or $R_{0}$ ) is not a universal constant but instead critically depends on structure of the host community.

\section{Vaccination}

A general vaccination strategy is one in which a substantial proportion of the population is vaccinated at random. The success of this measure depends on proportion (coverage), vaccine efficacy, and disease transmissibility. The availability of a vaccine, therefore, does not guarantee prevention unless both delivery and vaccine-induced immunity are sufficient. For example, Figure 5 shows that a mildly contagious disease like SARS may be thwarted by partial coverage $(\approx 75 \%)$ with a moderately efficacious vaccine ( $\approx 60 \%$ vaccine efficacy). Under this strategy, a moderately contagious disease can become epidemic unless a population receives $95 \%$ coverage with a $100 \%$ efficacious vaccine.

Ring vaccination of close contacts, on the other hand, is a very effective approach overall. This intervention, like quarantine, involves both transmission and contact reduction. Identifying the index patient results in a reduced infectious period. Subsequent identification and protection of his or her contacts through vaccination further limits the potential spread of the pathogen. Figure 5 considers the effect of ring vaccination on the population as a function of the effectiveness of patient isolation and the fraction of contacts that are successfully immunized. Partial protection of contacts may stem from inadequate contact tracing or an ineffective vaccine. For example, vaccinating $80 \%$ of

Mildly contagious disease Moderately contagious disease

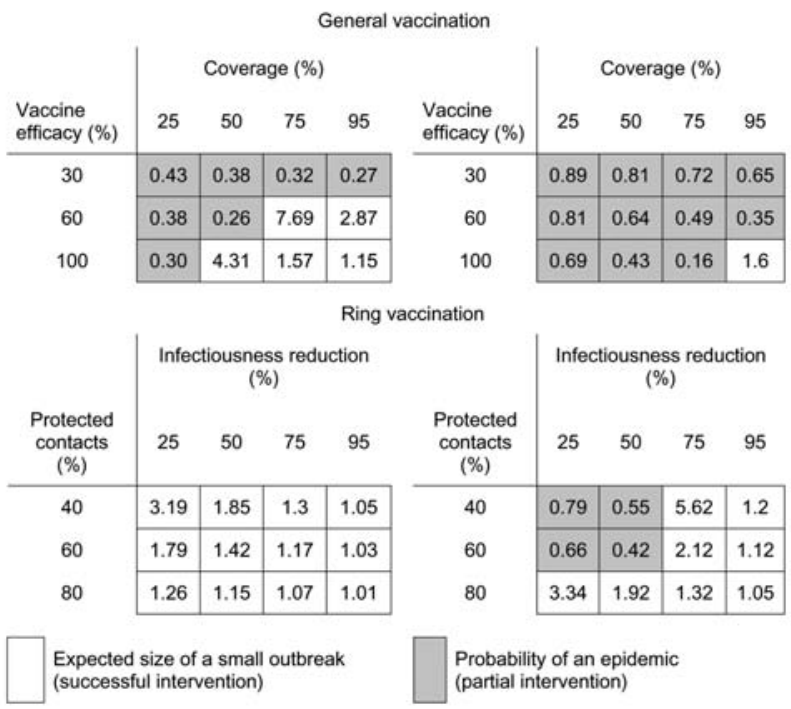

Figure 5. Comparing general vaccination and ring vaccination strategies. General vaccination protects a percentage of persons chosen randomly from the population with an efficacy determined by the vaccine itself. Ring vaccination involves isolating the patient (and the associated reduction in the infectious period) followed by targeted vaccination of contacts. The degree to which contacts are successfully protected depends on the success of contact tracing and the efficacy of the vaccine. See the Figure 3 caption for further details. 


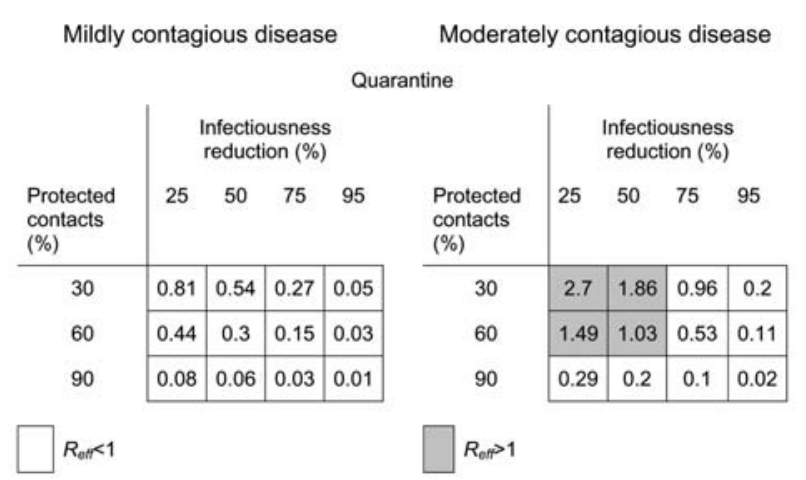

Figure 6. Intervention projections in terms of $R_{\text {eff. }}$ This figure presents the results in the lower panel of Figure 4 expressed in terms of effective reproductive number rather than the projected size of an outbreak. If $R_{\text {eff }}<1$ outbreaks will die out, while if $R_{\text {eff }}>1$, epidemics may ensue. Note that the shading indicates epidemic potential and coincides perfectly with the shading in Figure 4.

close contacts with a $50 \%$ efficacious vaccine is equivalent to vaccinating $40 \%$ of close contacts with a $100 \%$ efficacious vaccine. Ring vaccination can be a successful strategy for a mildly contagious disease with even a moderate surveillance infrastructure or a partially efficacious vaccine. However, ring vaccination requires more successful case identification, contact tracing, and vaccination when implemented against a highly contagious disease. Ring vaccination is only applicable to diseases with relatively long incubation periods that allow contacts to be identified, vaccinated, and develop a protective immune response. Thus, this strategy is more appropriate for diseases like smallpox (incubation period 12 days) than SARS (incubation period 2-7 days).

\section{Variation in Outbreak Size}

The white entries in Figures 3-5 report the expected (average) size of small outbreaks for diseases below the epidemic threshold. Any particular outbreak, however, may not be exactly equal to this average size. In the left panel of Figure 7, we show the average and standard deviation of outbreak sizes over the range of transmissibility values below the epidemic threshold. For each value of $T$, we estimate the standard deviation by using 1,000 simulated epidemics on the original urban network (without intervention). For low $T$, outbreaks tend to be small and close to the average outbreak size $s$. As $T$ increases toward the epidemic threshold, the distribution of outbreak sizes widens substantially, and $s$ becomes less informative. Given this variability, public health strategies should be based on bringing populations substantially under the epidemic threshold.

\section{Sensitivity Analysis}

Our mathematical predictions are based on a single simulated urban network with 2,000 households with an average of 2.6 people per household. To address the sensitivity of the predictions to the particular pattern of contacts in the network, we stochastically generated 100 urban networks of equal size and predicted the probability of an epidemic for the range of $T$ above the epidemic threshold. Since each of these 100 networks has a unique degree distribution, the value of the epidemic threshold varies. In particular we find that the average epidemic threshold is 0.04822 with a 95\% confidence interval of 0.04656-0.04988. Recall that the network used in the analysis above has an epidemic threshold $T_{c}=0.048$. The right panel of Figure 7 shows the mean probability of an epidemic across these 100 networks with 95\% confidence intervals. The probabilities for the particular network that we studied lie very close to the mean probabilities. The narrow confidence intervals suggest that our predictions are fairly robust to the particular architecture of the urban network. We further consider the effect of network size on these predictions in the online Appendix.

\section{Discussion}

Using contact network epidemiology, we evaluated various airborne infection control policies for a simulated urban setting like Vancouver. This approach explicitly captures the heterogeneous patterns of interpersonal contacts

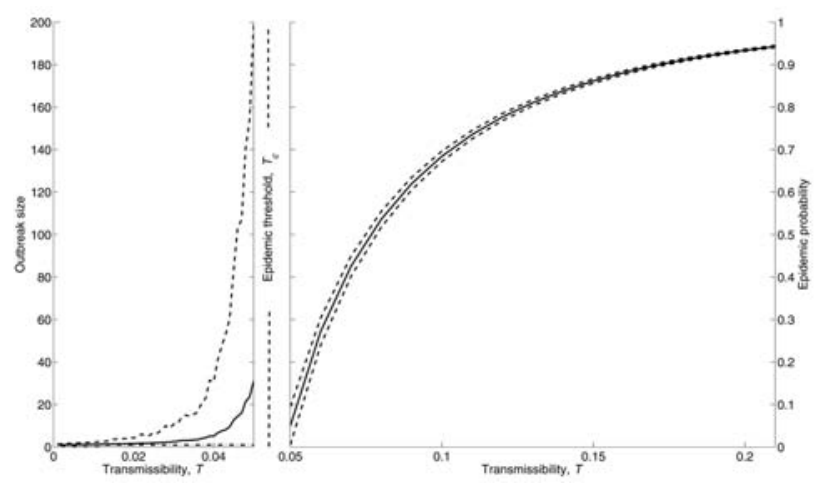

Figure 7. Left panel: variation of outbreak sizes as a function of transmissibility. We generated 1,000 epidemics for each of 20 values of $T$ from 0 to the epidemic threshold. The solid curve represents the mean of outbreak size $(\mu)$, the dashed curve represents 1 standard deviation above the mean $(\mu+\sigma)$, and the dotted line at the bottom shows the minimum size of an outbreak, which is always equal to 1 , meaning that after the introduction of the first infected case the disease did not spread further. Right panel: sensitivity of epidemic probability to network stochasticity. We generated 100 different networks, each with 2,000 households. Because of the stochastic nature of contact formation during network generation, these 100 networks contain different numbers and configurations of edges and therefore have different degree distributions. The solid curve shows the mean probability of an epidemic across the 100 networks for transmissibilities above the epidemic threshold, and the dashed curves are 95\% confidence limits for the mean probability of an epidemic. 
that lead to disease transmission and allows rapid mathematical prediction of the probability and distribution of an epidemic. This analysis does not depend on computationally intensive simulations. Furthermore, the approach allows one to quantitatively compare strategies that directly reduce the transmissibility of a pathogen or limit opportunities for a pathogen to spread. Although each strategy has been considered on its own, these methods can easily predict the effect of combined interventions for an entire spectrum of airborne infectious diseases, including SARS, smallpox, influenza, and meningococcal meningitis, among others.

Although the qualitative results of this analysis are applied to urban settings, the work is meant to be a proof of concept rather than to provide specific quantitative recommendations for urban control of communicable diseases such as SARS and smallpox. Until we have developed contact network models for a wide range of communities and assessed their generality, contact network epidemiology will need to be applied on a case-bycase basis. For example, hospitals can use these methods to improve control of nosocomial airborne infections. To start, each facility should model its particular network of patient-healthcare worker interactions, then calculate the effect of measures such as respiratory droplet precautions, grouping patients in cohorts, modifications to healthcare worker assignments, and vaccination (12).

The success of contact network epidemiology depends not only on realistic models of contact patterns but also on reliable estimates of the average transmissibility of the pathogen, $T$. As a respiratory pathogen begins to spread through a population, epidemiologists can rapidly identify the mode and rate of disease transmission. These data can provide critical input for intervention strategies. Historically, the rate of disease transmission has been measured and reported in terms of the basic reproductive number $R_{0}$, based on the doubling time of case counts in the early phase of an outbreak or epidemic. The value of $R_{0}$, however, may vary substantially, depending on the population in which it is measured. For example, recent estimates of $R_{0}$ for SARS ranged from 1.2 to 3.6 (34-36). In contrast, $T$ is not subject to the particular patterns of interaction within a community and can be reliably estimated in diverse settings. Measuring $T$ is only slightly more involved than measuring $R_{0}$. For each case, one must measure not just the number of secondary cases, but also the total number of contacts of the case-patient during the infectious period and then divide the first value by the second.

Just as enormous molecular and technological resources are often mobilized to develop vaccines and diagnostic tools for emerging infectious diseases of public health importance, we should also harness the powerful quantitative mathematical tools that help assess disease interventions. When an airborne pathogen strikes, public health officials should be able to make scientifically grounded decisions about the competing medical, economic, and social implications following deployment of control measures. We illustrate that contact network epidemiology can provide detailed and valuable insight into the fate and control of an outbreak. Integrating these tools into public health decision making should facilitate more rational strategies to manage emerging diseases, bioterrorist events, and pandemic influenza in situations in which empiric data are not yet available to guide decision making.

\section{Acknowledgments}

We thank Mark Newman for technical guidance.

This work was supported in part by grants from the Canadian Institutes of Health Research (CIHR) (FRN: 67803) to the British Columbia SARS Investigators Collaborative that includes B.P., L.A.M., D.M.S., M.K., D.M.P. and R.C.B. and from the National Science Foundation (DEB-0303636) to L.A.M. The Santa Fe Institute and CIHR supported the working visits of B.P. and L.A.M.

Dr. Pourbohloul is the director of the Division of Mathematical Modeling at the University of British Columbia Centre for Disease Control. He is leading a research group, funded by the CIHR, in the application of network theory to the prediction and control of SARS and other respiratory infections.

\section{References}

1. Poutanen SM, Low DE, Henry B, Finkelstein S, Rose D, Green K, et al. Identification of severe acute respiratory syndrome in Canada. $\mathrm{N}$ Engl J Med. 2003;348:1995-2005.

2. Brown H. WHO confirms human-to-human avian flu transmission. Lancet. 2004;363:462.

3. Reed KD, Melski JW, Graham MB, Regnery RL, Sotir MJ, Wegner $\mathrm{MV}$, et al. The detection of monkeypox in humans in the Western Hemisphere. N Engl J Med. 2004;350:342-50.

4. Nash D, Mostashari F, Fine A, Miller J, O’Leary D, Murray K, et al. The outbreak of West Nile virus infection in the New York City area in 1999. N Engl J Med. 2001;344:1807-14.

5. Normile D. Infectious diseases. First U.S. case of mad cow sharpens debate over testing. Science. 2004;303:156-7.

6. Swartz MN. Recognition and management of anthrax-an update. N Engl J Med. 2001;345:1621-6.

7. Charatan F. Widespread flu in United States exposes shortage of vaccine. BMJ. 2004;328:8.

8. Anderson RM, May RM. Infectious diseases of humans: dynamics and control. Oxford (UK): Oxford University Press; 1991.

9. Ferguson NM, Donnelly CA, Anderson RM. The foot-and-mouth epidemic in Great Britain: pattern of spread and impact of interventions. Science. 2001;292:1155-60.

10. Pourbohloul B, Rekart ML, Brunham RC. Impact of mass treatment on syphilis transmission: a mathematical modeling approach. Sex Transm Dis. 2003;30:297-305. 
11. Kaplan EH, Craft DL, Wein LM. Emergency response to a smallpox attack: the case of mass vaccination. Proc Natl Acad Sci U S A. 2002;99:10935-40.

12. Meyers LA, Newman ME, Martin M, Schrag S. Applying network theory to epidemics: control measures for Mycoplasma pneumoniae outbreaks. Emerg Infect Dis. 2003;9:204-10.

13. Newman MEJ. Spread of epidemic disease on networks. Phys Rev E Stat Nonlin Soft Matter Phys. 2002;66:016128.

14. Longini IM. A mathematical model for predicting the geographic spread of new infectious agents. Math Biosci. 1988;90:367.

15. Halloran ME, Longini IM Jr, Nizam A, Yang Y. Containing bioterrorist smallpox. Science. 2002;298:1428-32.

16. Sattenspiel L, Simon CP. The spread and persistence of infectious diseases in structured populations. Math Biosci. 1988;90:341.

17. Morris M. Data driven network models for the spread of disease. In: Mollison D, editor. Epidemic models: their structure and relation to data. Cambridge (UK): Cambridge University Press; 1995. p. 302-22.

18. Ball F, Mollison D, Scalia-Tomba G. Epidemics with two levels of mixing. The Annals of Applied Probability. 1997;7:46.

19. Diekmann O, de Jong MCM, Metz JAJ. A deterministic epidemic model taking account of repeated contacts between the same individuals. J Appl Probab. 1998;35:448-62.

20. Lloyd AL, May RM. Epidemiology. How viruses spread among computers and people. Science. 2001;292:1316.

21. Keeling MJ, Woolhouse MEJ, May RM, Davies G, Grenfell BT. Modeling vaccination strategies against foot-and-mouth disease. Nature. 2003;421:136-42.

22. Newman MEJ. The structure and function of complex networks. SIAM Review. 2003;45:167-256.

23. Statistics Canada [homepage on the Internet]. [cited 2004 May 6]. Available from http://www.statcan.ca

24. BC Statistics [homepage on the Internet]. [cited 2004 May 6]. Available from http://www.bcstats.gov.bc.ca

25. Centre for Health Services and Policy Research at University of British Columbia [homepage on the Internet]. [cited May 6]. Available from http://www.chspr.ubc.ca
26. City of Vancouver [homepage on the Internet]. [cited 2004 May 6]. Available from http://www.city.vancouver.bc.ca

27. Vancouver Public School Board [homepage on the Internet]. [cited 2004 May 6]. Available from http://www.vsb.bc.ca/default.htm

28. Diekmann O, Heesterbeek JAP. Mathematical epidemiology of infectious diseases: model building, analysis and interpretation. New York: John Wiley \& Sons; 2000.

29. Xu R-H, He J-F, Evans MR, Peng G-W, Field HE, Yu D-W, et al. Epidemiologic clues to SARS origin in China. Emerg Infect Dis. 2004;10:1030-7.

30. Yu IT, Li Y, Wong TW, Tam W, Chan AT, Lee JH, et al. Evidence of airborne transmission of the severe acute respiratory syndrome virus. N Engl J Med. 2004;350:1731-9.

31. Meyers LA, Pourbohloul B, Newman ME, Skowronski DM, Brunham RC. Network theory and SARS: predicting outbreak diversity. J Theor Biol. 2005;232:71-81.

32. Hethcote HW. The mathematics of infectious diseases. SIAM Review. 2000;42:599-653.

33. Bozzette SA, Boer R, Bhatnagar V, Brower JL, Keeler EB. A model for a smallpox-vaccination policy. N Engl J Med. 2003;348:416-25.

34. Chowell G, Fenimore PW, Castillo-Garsow MA, Castillo-Chavez C. SARS outbreaks in Ontario, Hong Kong, and Singapore: the role of diagnosis and isolation as a control mechanism. J Theor Biol. 2003;224:1-8.

35. Riley S, Fraser C, Donnelly CA, Ghani AC, Abu-Raddad LJ, Hedley AJ, et al. Transmission dynamics of the etiological agent of SARS in Hong Kong: impact of public health interventions. Science. 2003;300:1961-6.

36. Lipsitch M, Cohen T, Cooper B, Robins JM, Ma S, James L, et al. Transmission dynamics and control of severe acute respiratory syndrome. Science. 2003;300:1966-70.

Address for correspondence: Babak Pourbohloul, Division of Mathematical Modeling, University of British Columbia Centre for Disease Control, 655 West 12th Ave, Vancouver, British Columbia, V5Z 4R4 Canada; fax: 604-660-6066; email: babak.pourbohloul@bccdc.ca

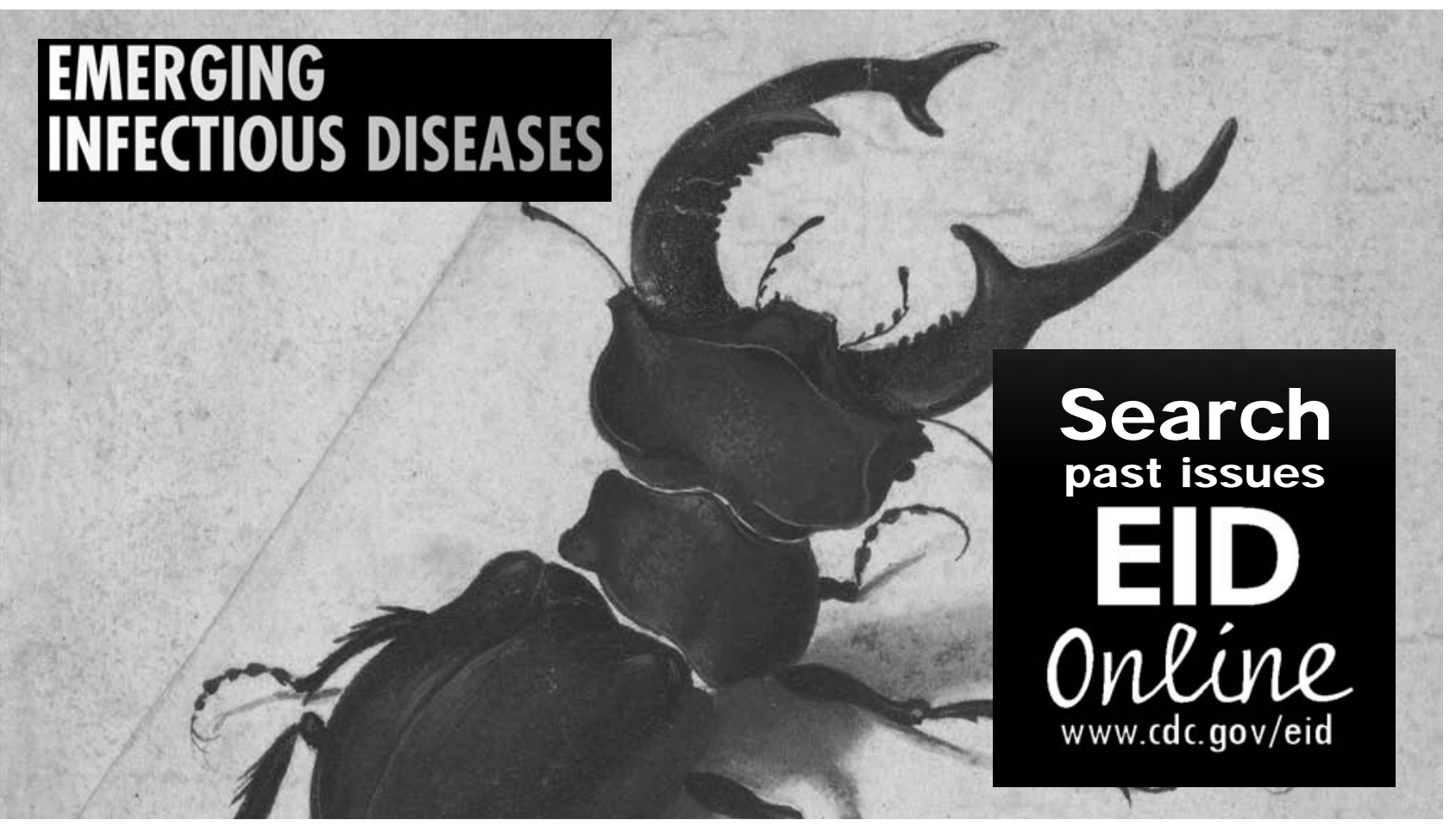

